\title{
Correction to: Majorization and the spectral radius of starlike trees
}

\section{Mohammad Reza Oboudi ${ }^{1,2}$}

\section{Correction to: J Comb Optim https://doi.org/10.1007/s10878-018-0287-5}

The original version of the article contained a mistake. In page 2, second paragraph, line 7, the word "and" was inadvertently missed. The corrected sentence is "For example $T\left(n_{1}\right) \ldots . t \leq k-1$ and $n_{t+1}, \ldots, n_{k} \geq 2, \ldots$. .

The original article has been corrected.

The original article can be found online at https://doi.org/10.1007/s10878-018-0287-5.

\. Mohammad Reza Oboudi

mr_oboudi@shirazu.ac.ir; mr_oboudi@yahoo.com

1 Department of Mathematics, College of Sciences, Shiraz University, Shiraz 71457-44776, Iran

2 School of Mathematics, Institute for Research in Fundamental Sciences (IPM), P.O. Box 19395-5746, Tehran, Iran 